\title{
Fatores motivacionais e contribuições das mobilidades internacionais de estudantes de uma instituição federal do Rio Grande do Sul
}

\author{
Motivational factors and contributions of international mobilities of students \\ from a federal institution of Rio Grande do Sul
}

Factores motivacionales y contribuciones de las movilidades internacionales de estudiantes de una institución federal de Rio Grande do Sul

\author{
ANA PAULA PERLIN \\ GABRIELA ROSSATO \\ FRANCIES DIEGO MOTKE \\ CLANDIA MAFFINI GOMES \\ JORDANA MARQUES KNEIPP
}

\begin{abstract}
Resumo: Este estudo foi desenvolvido com o objetivo de identificar as motivações e contribuições da mobilidade acadêmica internacional para os alunos de uma Instituição Federal do Rio Grande do Sul. Por meio dos resultados apresentados, foi possível identificar os fatores que motivaram a realização de intercâmbio pelos estudantes, os quais parecem estar mais relacionados a experiências pessoais quando comparados com objetivos profissionais. Entre as razões que motivaram a escolha do país de destino do intercâmbio, percebeu-se que fatores relacionados à estrutura que o país de destino oferece, com baixos custos, boa infraestrutura e instituições acadêmicas atraentes, são razões significativas para a escolha do país de destino do estudante em mobilidade acadêmica da instituição analisada.
\end{abstract}

Palavras-chave: Mobilidade Acadêmica. Fatores Motivacionais.

\begin{abstract}
This study was developed with the objective of identifying the motivations and contributions of international academic mobility for the students of a Federal Institution of Rio Grande do Sul. Through the presented results, it was possible to identify the factors that motivated the exchange of students, which seem to be more related to personal experiences when compared to professional goals. Among the reasons that led to the choice of the destination country of the exchange, factors related to the structure that the country of origin offers, with low costs, good infrastructure and attractive academic institutions, are significant reasons for choosing the country of destination of the students in academic mobility of the analyzed institution.
\end{abstract}

Keywords: Academic Mobility, Motivational Factors.

RBPAE - v. 34, n. 3, p. 775 - 794, set./dez. $2018 \cdot 775$ 
Resumen: Este estudio fue desarrollado con el objetivo de identificar las motivaciones y contribuciones de la movilidad académica internacional para los alumnos de una Institución Federal de Rio Grande do Sul. A través de los resultados presentados, fue posible identificar los factores que motivaron la realización de intercambio por los estudiantes, los cuales parecen estar más relacionados con experiencias personales cuando comparados objetivos profesionales. Entre las razones que motivaron la elección del país de destino del intercambio, se percibió que factores relacionados con la estructura que el país de origen ofrece, con bajos costos, buena infraestructura e instituciones académicas atractivas, son razones significativas para la elección del país de destino del estudiante en movilidad académica de la institución analizada.

Palabras clave: Movilidad Académica. Factores motivacionales.

\section{INTRODUÇÃO}

A redução das barreiras nacionais, os avanços dos meios de comunicação e do sistema produtivo são fatores que impulsionaram o processo de internacionalização das instituições. A internacionalização, proporcionou a inserção de muitos negócios no contexto internacional e a expansão do conhecimento e de redes de compartilhamento, tanto em instituições empresariais quanto em instituições acadêmicas.

Segundo Stallivieri (2004), a internacionalização das instituições de ensino superior, utilizando-se de métodos cooperativos, estão proporcionando melhorias na qualidade do ensino e da pesquisa. Ambos, quando trabalhados juntos, geram condições para o desenvolvimento dos países e para o aumento da qualidade de vida da população.

Ainda de acordo com, Stallivieri (2004) "a universidade, como santuário de desenvolvimento da pesquisa e da produção do conhecimento científico, tem a vocação de ser internacional, pois o conhecimento não tem nacionalidade" (STALLIVIERI, 2004, p. 40).

Conforme Van Damme (2001), os desafios resultantes da internacionalização estão empurrando universidades para desenvolver novas formas de esforços e políticas de internacionalização. No contexto acadêmico, Van Damme (2001) aborda algumas formas de internacionalização, como: mobilidade estudantil, mobilidade de docentes, internacionalização de currículos, abertura de filiais, cooperação institucional e de rede, acordo de reconhecimento mútuo, redes transnacionais de universidades e educação superior virtual transnacional.

Para Castro e Neto (2012), embora a mobilidade estudantil não seja um fenômeno novo, ela é restruturada na contemporaneidade em função do processo de globalização e das atuais estratégias de internalização do ensino superior. Os 
autores afirmam que a mobilidade acadêmica envolve uma amplitude de fatores, não apenas o movimento de deslocamento territorial; envolve aspectos sociais, estruturas, meios, culturas e significados.

Desse modo, o presente estudo tem como objetivo identificar as motivações e contribuições da mobilidade acadêmica internacional para os alunos de uma instituição federal do Rio Grande do Sul.

Além da introdução, este trabalho está organizado em mais quatro seções. A segunda se refere à contextualização da internacionalização no âmbito da mobilidade acadêmica internacional. A terceira seção trata especificamente dos aspectos metodológicos utilizados para o desenvolvimento do estudo. A quarta apresenta a análise dos dados e discussão dos resultados, e, por fim, as considerações finais.

\section{REFERENCIAL TEÓRICO}

O objetivo do referencial teórico é apresentar a fundamentação teórica essencial ao desenvolvimento do trabalho, por meio da síntese da literatura relacionada com os diversos aspectos do tema estudado. Assim, neste capítulo, serão apresentados definições e conceitos sobre a temática Mobilidade Acadêmica Internacional.

\section{MOBILIDADE ACADÊMICA INTERNACIONAL}

A troca de conhecimentos e experiências entre as universidades possibilita à Instituição e ao aluno participar da mundialização, que interfere na educação e colabora para que se abram as portas da Universidade para novos conhecimentos, novas culturas e formas de gestão (GUIMARÃES, S. R. E. F; TADEUCCI, M. DE. S.; OLIVEIRA, DE. A. L, 2013).

Corroborando, Romêo (2003) afirma que as universidades sempre foram instituições internacionais e devem ser palco de diálogo internacional, garantindo o progresso científico e tecnológico. Nesse sentido, a mobilidade estudantil e outras formas de intercâmbio e contato podem ajudar as universidades a desempenhar seu papel na sociedade, sendo a mobilidade de alunos, servidores e funcionários imprescindível na modernização das universidades da região.

A mobilidade internacional é fomentada, segundo Nogueira et. al (2008) pelo crescente número de parcerias entre universidades, acordos bilaterais e programas institucionais. As oportunidades de mobilidade internacional 
existentes para os estudantes parecem, assim, estar cada vez mais acessíveis, sendo de responsabilidade dos estudantes optar segundo seus critérios e preferências por um país de destino.

De acordo com Lima e Riegel (2010), a mobilidade de estudantes configura um tema incluído na agenda internacional dos pesquisadores, os quais buscam compreender e localizar os aspectos que influenciam verdadeira legião de jovens a decidir por uma formação internacional.

Em seus estudos, Lima e Riegel (2010) buscaram conhecer as motivações que justificam o crescente interesse dos estudantes de administração pelos programas de formação internacional. Como principais contribuições percebem que a principal razão que norteou a escolha do país de destino da mobilidade dos estudantes, foi a questão da língua inglesa. Esse fato vem ao encontro de Nogueira et. al (2008), os quais afirmam que há uma preferência evidente por certos países, visto que a maioria relativa dos jovens matriculados em instituições universitárias fora de seu país de origem encontra como destino os Estados Unidos.

Conforme Lima e Riegel (2010), alguns autores vêm trazendo importantes contribuições para a discussão das principais motivações dos estudantes quando se referem a mobilidade internacional; são eles: Kurt Larsen e Stéphan VincentLancrin (2002); Marie-Claude Muñoz (2004); Mohamed Harfi (2004); Mohamed Harfi e Claude Mathieu (2006). A partir desses autores, é possível dividir as motivações em quatro tipos: motivações de ordem sociocultural, acadêmica, econômico-comercial e político-administrativo.

As motivações socioculturais se referem, segundo esses autores, a questões como Língua oficial do país de destino, proximidade geográfica e cultural entre o país de origem e de destino, ligações históricas pré-existentes, presença de grupos de referência e qualidade de vida e atratividade cultural existente no país de destino: estabilidade política e segurança pública. Já as motivações acadêmicas se referem, geralmente, às limitações na oferta de programas e cursos no sistema de educação do país de origem, equivalência de diplomas, possibilidade de estudantes internacionais terem acesso aos cursos desejados no país de destino, reputação e percepção de qualidade do sistema educativo, existência de programas bi/multilaterais e existência de política de bolsa de estudo e validação do diploma.

Os autores ainda incluem as motivações econômico-comerciais, referentes às ligações econômicas pré-existentes de cooperação, custo de vida no país de destino, comparação entre os custos financeiros envolvidos, existência e acesso à infraestrutura política de financiamento da mobilidade estudantil (concessão de bolsas ou de estágio remunerado), seguro de saúde, alojamento para estudante, restaurante universitário, oferta de curso da língua, valorização das competências desenvolvidas pelas instituições do país de destino, valor dos 
diplomas expedidos pelo país de destino no mercado de trabalho, possibilidade de trabalho durante seus estudos e possibilidade de permanecer no país de destino após o término do curso. $\mathrm{Na}$ quarta categoria, os autores incluem os aspectos políticos administrativos, relacionados à política de imigração que facilite a obtenção de visto de estudante no país de destino.

Conforme o Relatório Mundial da UNESCO (2006), as políticas educacionais podem intervir no crescimento ou no declínio da diversidade cultural e "devem promover a educação pela e para a diversidade".

Segundo Stallivieri (2004), a internacionalização das instituições de ensino superior, utilizando de métodos cooperativos, vem proporcionando melhorias na qualidade do ensino e da pesquisa. Ambos, quando trabalhados juntos, geram condições para o desenvolvimento dos países e para o aumento da qualidade de vida da população.

Ainda de acordo com Stallivieri (2004), "A universidade, como santuário de desenvolvimento da pesquisa e da produção do conhecimento científico, tem a vocação de ser internacional, pois o conhecimento não tem nacionalidade" (STALLIVIERI, 2004, p. 40).

$O$ fenômeno da internacionalização no setor educacional acontece, de acordo com Lima e Maranhão (2009), de dois modos: primeiro, pela internacionalização ativa, ou seja, quando os países mantêm políticas de Estado voltadas para atração e acolhimento acadêmico e, segundo, pela internacionalização passiva, que pode ser caracterizada como uma política pouco criteriosa para envio dos estudantes ao exterior, bem como a fraca capacidade de acolhimento e a oferta de serviços educacionais do país.

Para Ianni (2005), o Estado, as empresas e a família passaram a valorizar as instituições de ensino superior que incentivam pesquisas e buscam formar profissionais de sucesso. Segundo Filippetti (2007), a capitalização de recursos financeiros diretos e indiretos e as diversas vantagens dos países que atraem e acolhem os estudantes contribuem para ampliar a rede mundial de influência cultural e política do país, bem como beneficiar-se de mão de obra especializada e promover transferência de tecnologia.

Assim, os conceitos de mobilidade acadêmica internacional sofreram mudanças no decorrer dos anos. Uma definição importante sobre o tema é da UNESCO (2006), que divide a mobilidade em vertical e horizontal. A mobilidade vertical é aquela que presume que estudantes vindos de países menos favorecidos economicamente deslocam-se para países financeiramente mais estruturados com o objetivo de ter acesso a programas de alto rendimento e, consequentemente, melhores colocações do mercado de trabalho. Já a mobilidade horizontal é realizada em períodos de curto prazo, e as instituições envolvidas são vistas 
em nível de igualdade de ensino. Dessa forma, os estudantes têm uma rápida adaptação ao local e adquirem os mesmos benefícios de uma mobilidade vertical (UNESCO, 2006).

A língua falada no país é um ponto de extrema relevância na escolha do destino para o estudante. Afim de comprovar, no ano de 2006 foram divulgados os seis destinos mais procurados pelos estudantes internacionais, sendo eles: Estados Unidos (22\%), Reino Unido (11\%), Alemanha (10\%), França (9\%) e Austrália (6\%) (UNESCO, 2008).

$\mathrm{O}$ interesse em aprender a língua inglesa é também demonstrado nos resultados dos estudos realizados no Brasil por Aguiar (2009), Nogueira et al., (2008; 2004); Douek e Zylberstajn (2007), Almeida (2004), Prado (2004), entre outros.

Tabela no.1 - Principais Países Receptores de Estudantes (2001-2006)

\begin{tabular}{|c|c|c|c|c|c|c|}
\hline PAÍSES & 2001 & $\mathbf{2 0 0 2}$ & $\mathbf{2 0 0 3}$ & $\mathbf{2 0 0 4}$ & $\mathbf{2 0 0 5}$ & $\mathbf{2 0 0 6}$ \\
\hline $\begin{array}{c}\text { ESTADOS } \\
\text { UNIDOS }\end{array}$ & 475169 & 582996 & 582996 & 572509 & 590128 & 584814 \\
\hline $\begin{array}{c}\text { REINO } \\
\text { UNIDO }\end{array}$ & 225722 & 225722 & 227273 & 300056 & 318399 & 330078 \\
\hline ALEMANHA & 199132 & 219039 & 240619 & 260314 & 259797 & 259797 \\
\hline FRANÇA & 147402 & 147402 & 221567 & 237587 & 236518 & 247510 \\
\hline AUSTRÁLIA & 105764 & 120987 & 179619 & 166954 & 207264 & 207264 \\
\hline CANADÁ & 40033 & 40033 & 40033 & 40033 & 132982 & 75546 \\
\hline
\end{tabular}

Fonte: Recueil de Données Mondiales sur l'Education. Institut de Statistique/UNESCO, 2003, 2004, 2005, 2006, 2007.

$\mathrm{Na}$ América latina, a situação é parecida; levando-se em consideração o ano de 2006, entre os seis países latino-americanos que mais enviaram estudantes para o exterior, a maioria tem como primeiro destino os Estados Unidos (UNESCO, 2008).

\section{MÉTODO DO ESTUDO}

O estudo desenvolvido caracteriza-se, quanto aos objetivos, como descritivo, uma vez que este tipo de estudo pretende descrever os fatos e fenômenos de determinada realidade (TRIVINOS, 1987). Quanto à abordagem, este estudo caracteriza-se como quantitativo. De acordo com o Richardson (1999), o método quantitativo caracteriza-se pelo emprego da quantificação na coleta e tratamento das informações por meio de técnicas estatísticas. 
Utilizou-se o método survey, que é um método quantitativo que busca a obtenção de dados ou informações sobre características, ações ou opiniões de determinado grupo de pessoas, indicado como representante de uma populaçãoalvo, por meio de um instrumento de pesquisa, normalmente um questionário (PINSONNEAULT; KRAEMER,1993).

Para identificar as motivações e contribuições da mobilidade acadêmica internacional, escolheu-se como objeto de estudo os estudantes de uma Universidade Federal do Rio Grande do Sul que participaram de mobilidade acadêmica no exterior.

Como instrumento de coleta de dados, trabalhou-se com um questionário estruturado composto por perguntas fechadas. $\mathrm{O}$ instrumento de coleta de dados foi adaptado do estudo desenvolvido por Lima e Riegel (2010).

O questionário foi dividido em duas partes. A primeira buscou caracterizar o perfil dos estudantes que participaram de mobilidade acadêmica. A segunda parte procurou identificar os objetivos que motivaram o investimento no programa de intercâmbio, as razões que motivaram a escolha do país de destino do intercâmbio e os resultados percebidos com o programa de intercâmbio, com base em uma escala likert de sete pontos, sendo (1) discordo totalmente até (7) concordo totalmente.

A coleta de dados foi realizada em setembro e outubro de 2017. Os questionários foram aplicados de forma eletrônica através da ferramenta Google Drive e se obteve um retorno de 60 estudantes da Universidade Federal de Santa Maria. A amostra caracteriza-se como não- probabilística e por acessibilidade.

Posteriormente, os dados foram tabulados em uma planilha eletrônica, onde se utilizou o programa Microsoft Excel 2016, e analisados com o auxílio do Statistical Package for the Social Sciences (SPSS) 23 que permite análises estatísticas. Os resultados são apresentados em forma de tabelas, analisados individualmente pelos pesquisadores e descritos em forma de texto, na próxima sessão.

\section{ANÁLISE E DISCUSSÃO DOS RESULTADOS}

Os resultados da pesquisa são apresentados da seguinte forma: caracterização da amostra, análise dos objetivos do intercâmbio, razões da escolha do país, análise dos resultados percebidos, análise das variáveis por gênero, análise das variáveis por faixa etária e análise das variáveis por renda familiar. A seguir, apresenta-se a caracterização dos respondentes. 


\section{CARACTERIZAÇÃO DA AMOSTRA}

Evidencia-se que, entre os respondentes, a maior parcela corresponde ao gênero feminino $(71,7 \%)$, enquanto que o gênero masculino corresponde a $28,3 \%$ dos investigados. Além disso, a faixa etária dos estudantes analisados concentra-se, principalmente, entre 22 e 23 anos (51,7\%), além de uma parcela de $25 \%$ que têm entre 24 e 25 anos e outros 13,3\% que têm 21 anos ou menos. Quando se analisa o estado civil, a grande maioria dos estudantes que realizaram mobilidade acadêmica são solteiros (93,3\%), sendo somente 5\% em união estável e 1,7\% casado. Em relação a faixa de renda, percebe-se que a renda familiar desses estudantes concentra-se, principalmente, nas classes $\mathrm{C}$ (de 3 a 5 salários mínimos) - 35\%, e B (de 5 a 15 salários mínimos) 30\%. A classe mais alta (mais de 15 salários mínimos) corresponde a $15 \%$ dos estudantes que realizaram mobilidade acadêmica, enquanto as classes mais baixas correspondem a 20\% (de 1 a 3 salários mínimos) - 18,3\% e até 1 salário mínimo - 1,7\%. Percebe-se, também, que a preferência para realização do intercâmbio corresponde ao período da metade para o fim da graduação, entre o $5^{\circ}$ e o $8^{\circ}$ semestre, para $85 \%$ dos respondentes, sendo $26,7 \%$, no $5^{\circ}$ semestre, $23,3 \%$ no $7^{\circ}$ semestre, $21,7 \%$ no $6^{\circ}$ semestre e $13,3 \%$ no $8^{\circ}$ semestre. Quanto ao curso dos graduandos investigados, destacam-se cursos pertencentes ao Centro de Ciências Sociais e Humanas (CCSH) da universidade, entre eles: jornalismo (11,7\%), relações internacionais $(11,7 \%)$, direito $(6,7 \%)$, administração $(5 \%)$ e ciências econômicas ( $5 \%$ ). Os países mais procurados para realização da mobilidade acadêmica entre os investigados são os países europeus $(49,1 \%)$, entre eles: Portugal $(23,7 \%)$, Espanha (11,9\%), Alemanha $(6,8 \%)$ e Inglaterra (5,1\%). Os sul-americanos (35,6\%), entre eles Argentina (18,6\%), Chile $(8,5 \%)$, Colômbia $(3,4 \%)$ e Paraguai $(3,4 \%)$. Além desses, o norte-americano México $(11,9 \%)$. O tempo médio de permanência corresponde, majoritariamente, entre 3 e 6 meses $(80 \%)$.

A Tabela 1, a seguir, apresenta os dados representativos da amostra dos estudantes que realizaram mobilidade acadêmica. 
Tabela 1 - Caracterização da amostra

\begin{tabular}{|c|c|c|c|}
\hline \multicolumn{2}{|l|}{ Gênero } & \multicolumn{2}{|c|}{ Semestre do curso em que realizou } \\
\hline Masculino & $17(28,3 \%)$ & $3^{\circ}$ Semestre & $1(1,7 \%)$ \\
\hline Feminino & $43(71,7 \%)$ & $4^{\circ}$ Semestre & $2(3,3 \%)$ \\
\hline Faixa Etária & & $5^{\circ}$ Semestre & $16(26,7 \%)$ \\
\hline Até 21 anos & $8(13,3 \%)$ & $6^{\circ}$ Semestre & $13(21,7 \%)$ \\
\hline 22 a 23 anos & $31(51,7 \%)$ & $7^{\circ}$ Semestre & $14(23,3 \%)$ \\
\hline 24 a 25 anos & $15(25 \%)$ & $8^{\circ}$ Semestre & $8(13,3 \%)$ \\
\hline De 26 a 29 anos & $3(5 \%)$ & $9^{\circ}$ Semestre & $2(3,3 \%)$ \\
\hline 30 anos ou mais & $3(5 \%)$ & $10^{\circ}$ Semestre & $2(3,3 \%)$ \\
\hline \multicolumn{2}{|l|}{ Estado Civil } & Graduado(a) & $2(3,3 \%)$ \\
\hline Solteiro(a) & $56(93,3 \%)$ & \multicolumn{2}{|l|}{ País em que realizou } \\
\hline Casado(a) & $1(1,7 \%)$ & Portugal & $14(23,7 \%)$ \\
\hline União estável & $3(5 \%)$ & Argentina & $11(18,6 \%)$ \\
\hline \multicolumn{2}{|l|}{ Renda Familiar } & Espanha & $7(11,9 \%)$ \\
\hline Até 1 salário mínimo & $1(1,7 \%)$ & México & $7(11,9 \%)$ \\
\hline De 1 a 3 salários mínimos & $11(18,3 \%)$ & Chile & $5(8,5 \%)$ \\
\hline De 3 a 5 salários mínimos & $21(35 \%)$ & Alemanha & $4(6,8 \%)$ \\
\hline De 5 a 15 salários mínimos & $18(30 \%)$ & Inglaterra & $3(5,1 \%)$ \\
\hline Mais de 15 salários mínimos & $9(15 \%)$ & Colômbia & $2(3,4 \%)$ \\
\hline \multicolumn{2}{|l|}{ Curso } & Paraguai & $2(3,4 \%)$ \\
\hline Jornalismo & $7(11,7 \%)$ & Estados Unidos & $1(1,7 \%)$ \\
\hline Relações Internacionais & $7(11,7 \%)$ & Grécia & $1(1,7 \%)$ \\
\hline Direito & $4(6,7 \%)$ & Porto Rico & $1(1,7 \%)$ \\
\hline Administração & $3(5 \%)$ & Uruguai & $1(1,7 \%)$ \\
\hline Ciências Econômicas & $3(5 \%)$ & \multicolumn{2}{|c|}{ Tempo médio de permanência } \\
\hline Arquitetura e Urbanismo & $2(3,3 \%)$ & De 1 a 3 meses & $1(1,7 \%)$ \\
\hline Educação Física & $2(3,3 \%)$ & De 3 a 6 meses & $48(80 \%)$ \\
\hline Engenharia Ambiental e Sanitária & $2(3,3 \%)$ & De 6 a 9 meses & $8(13,3 \%)$ \\
\hline Engenharia de Computação & $2(3,3 \%)$ & \multirow[t]{8}{*}{ De 9 meses a 1 ano } & $3(5 \%)$ \\
\hline Engenharia Florestal & $2(3,3 \%)$ & & \\
\hline Engenharia Mecânica & $2(3,3 \%)$ & & \\
\hline História & $2(3,3 \%)$ & & \\
\hline Letras & $2(3,3 \%)$ & & \\
\hline Odontologia & $2(3,3 \%)$ & & \\
\hline Psicologia & $2(3,3 \%)$ & & \\
\hline Outros & $16(26,7 \%)$ & & \\
\hline
\end{tabular}

Fonte: Elaboração dos autores. 
A partir da análise da Tabela 1, percebe-se que, de forma geral, a amostra representa os estudantes que realizaram mobilidade acadêmica, em sua maioria, do sexo feminino, com idade entre 22 e 23 anos, solteiras, com renda entre 3 a 15 salários mínimos, com intercâmbio realizado entre o $5^{\circ}$ e o $8^{\circ}$ semestre do curso, pertencentes aos cursos do Centro de Ciências Sociais e Humanas (CCSH) da instituição, para países europeus ou americanos, com duração de 3 a 6 meses.

A seguir, apresenta-se a análise dos objetivos de realização do intercâmbio, das razões de escolha do país de destino e dos resultados percebidos.

\section{ANÁLISE DOS OBJETIVOS DO INTERCÂMBIO, RAZÕES DA ESCOLHA DO PAÍS E RESULTADOS PERCEBIDOS}

Primeiramente, analisam-se os objetivos que motivaram o investimento no programa de intercâmbio. Posteriormente, avaliam-se as razões que motivaram a escolha do país de destino do intercâmbio. Por fim, especulam-se os resultados percebidos com o programa de intercâmbio.

$\mathrm{Na}$ Tabela 2, a seguir, apresentam-se a média e o desvio padrão para cada variável analisada.

\section{Tabela 2 - Objetivos do intercâmbio, razões da escolha do país e resultados percebidos}

\begin{tabular}{|l|c|c|}
\hline Objetivos que motivaram o investimento no programa de intercâmbio & Média & Desvio Padrão \\
\hline Vivência pessoal & 6,62 & 0,993 \\
\hline Conhecer outra cultura & 6,28 & 1,462 \\
\hline Conhecer outro país & 6,28 & 1,354 \\
\hline Desenvolver competências relacionadas à profissão & 6,00 & 1,221 \\
\hline Aperfeiçoar o conhecimento de língua estrangeira & 5,67 & 2,039 \\
\hline Prospectar alternativas de pós-graduação & 5,02 & 1,944 \\
\hline Prospectar alternativa de estágio internacional e remunerado & 3,67 & 2,113 \\
\hline Razões que motivaram a escolha do país de destino do intercâmbio & Média & Desvio Padrão \\
\hline Custos envolvidos & 5,10 & 1,920 \\
\hline Boa infraestrutura de acolhimento & 5,03 & 1,966 \\
\hline Instituições reconhecidas no mercado de trabalho & 4,95 & 2,045 \\
\hline Oferta de atividades compatíveis com o nível intelectual dos estudantes & 4,83 & 1,950 \\
\hline Facilidade de obtenção de visto & 4,10 & 2,268 \\
\hline Estabilidade da moeda local & 3,58 & 1,960 \\
\hline Destino aprovado pelos pais & 3,45 & 2,432 \\
\hline Indicação de amigos & 3,22 & 2,140 \\
\hline
\end{tabular}




\section{Tabela 2 - Objetivos do intercâmbio, razões da escolha do país e resultados percebidos}

\begin{tabular}{|l|c|c|}
\hline Razões que motivaram a escolha do país de destino do intercâmbio & Média & Desvio Padrão \\
\hline Possibilidade de compatibilizar estudo e trabalho & 2,32 & 2,087 \\
\hline Proximidade geográfica com o Brasil & 2,17 & 1,924 \\
\hline Ter como língua oficial o inglês & 1,90 & 1,811 \\
\hline Indicação da agência & 1,57 & 1,280 \\
\hline Resultados percebidos com o programa de intercâmbio & Média & Desvio Padrão \\
\hline Vivência pessoal & 6,68 & 0,948 \\
\hline Conhecimento de outro país & 6,63 & 0,863 \\
\hline Conhecimento de outra cultura & 6,38 & 1,342 \\
\hline Melhoramento do currículo & 6,13 & 1,241 \\
\hline Aperfeiçoamento de uma segunda língua & 5,73 & 1,965 \\
\hline Desenvolvimento de competências relacionadas à profissão & 5,70 & 1,555 \\
\hline Prospecção de alternativas de pós-graduação & 4,87 & 2,071 \\
\hline Prospecção de alternativa de estágio internacional e remunerado & 3,55 & 2,197 \\
\hline
\end{tabular}

Fonte: Elaboração dos autores.

Quanto aos objetivos que motivaram os estudantes da instituição analisada a optarem pela inserção em um programa de intercâmbio, destaca-se, principalmente, a 'vivência pessoal' $(6,62)$, ou seja, a oportunidade de viver em um país diferente do de sua origem, de forma a agregar uma experiência de vida. Outros objetivos que se destacaram foram 'conhecer outra cultura' $(6,28)$ e 'conhecer outro país' $(6,28)$, ou seja, os estudantes optaram pela realização de mobilidade acadêmica para vivenciar uma cultura diferente e para ter a oportunidade de viajar para um país ainda não visitado.

Outro fator com média representativa diz respeito a 'desenvolver competências relacionadas à profissão’ $(6,00)$, o que nos remete ao fato que, para os estudantes analisados, a inserção no programa de intercâmbio foi motivada, também, por questões relacionadas ao curso que optaram por seguir. Além disso, 'aperfeiçoar o conhecimento de língua estrangeira' $(5,67)$ também está entre os principais motivos da realização de mobilidade acadêmica, visto que entre os países preferidos estão, em sua maioria, aqueles em que o idioma oficial não é o português. Além disso, destaca-se que 'prospectar alternativas de pósgraduação’ (5,02) também está entre os objetivos do intercâmbio, indicando que o intercâmbio é percebido, inclusive, como uma experiência capaz de proporcionar maior amplitude de oportunidades para aperfeiçoamento profissional. 
A variável com menor média entre os analisados foi 'prospectar alternativa de estágio internacional e remunerado' $(3,67)$, revelando que participar de programas de estágio em empresas no exterior não era objetivo relevante para a maioria dos estudantes em mobilidade acadêmica, comparado aos demais motivos relatados.

A partir destas evidências, percebe-se que os principais objetivos que motivaram a realização de intercâmbio por parte de estudantes da instituição analisada estão mais relacionados a experiências pessoais, se comparados com experiências profissionais.

Quanto às razões que motivaram a escolha do país de destino do intercâmbio, destaca-se, principalmente, o custo-benefício oferecido pelo local, ou seja, baixo 'custo envolvido' $(5,10)$ e 'boa infraestrutura de acolhimento' $(5,03)$.

Algumas questões que se destacaram também na escolha do país de destino são a existência de 'instituições reconhecidas no mercado de trabalho' $(4,95)$ e a 'oferta de atividades compatíveis com o nível intelectual dos estudantes' $(4,83)$, indicando que a instituição acadêmica e as atividades que ela proporciona têm um impacto significativo na escolha do país de destino.

As razões que apresentaram baixo impacto para a maioria dos estudantes analisados foram 'indicação da agência' $(1,57)$, 'ter como língua oficial o inglês' (1,90), 'proximidade geográfica com o Brasil' $(2,17)$ e 'possibilidade de compatibilizar estudo e trabalho’ $(2,32)$. Esses resultados indicam que a razão de escolha do país de destino diz respeito a que, em grande parte, para a opção do estudante, não há uma preferência por aperfeiçoamento do idioma inglês, visto que grande parte dos países escolhidos tem como idioma principal o espanhol; não há preferência por uma localização geográfica próxima ao país de origem, visto que grande parte dos países de destino são localizados no continente europeu, e não há significativa preferência por compatibilizar estudo e trabalho na escolha do país, posto que a possibilidade de estágio internacional não está entre os objetivos mais relevantes para os estudantes analisados.

Diante dessa análise, percebe-se que fatores relacionados às condições que o país de origem oferece, como baixos custos, boa infraestrutura e instituições acadêmicas atraentes, são razões significativas para a escolha do país de destino do estudante em mobilidade acadêmica da instituição analisada.

Por fim, quanto aos resultados percebidos com o programa de intercâmbio, destacam- se a 'vivência pessoal' $(6,68)$, o 'conhecimento de outro país' $(6,63)$ e o conhecimento de outra cultura $(6,38)$. Esses resultados corroboram os principais objetivos destacados pelos estudantes analisados, indicando que a experiência pessoal pretendida com o programa de mobilidade acadêmica foi vivenciada. 
Outros resultados que podem ser destacados dizem respeito ao 'melhoramento do currículo' (6,13), ao 'aperfeiçoamento de uma segunda língua' $(5,73)$, ao ‘desenvolvimento de competências relacionadas à profissão’ $(5,70)$ e, em menor grau, a 'prospecção de alternativas de pós-graduação' (4,87). Esses resultados, juntamente com a 'prospecção de alternativa de estágio internacional e remunerado' $(3,55)$, que obteve a menor média entre os analisados, confirmam, também, que os objetivos menos relevantes para os acadêmicos no intercâmbio apresentaram os resultados menos significativos quando comparados aos demais, apesar de que a maioria deles podem ser considerados satisfatórios.

Logo, em geral, os resultados mais significativos percebidos pelos estudantes com o programa de intercâmbio, para a amostra analisada, estão relacionados às competências pessoais, confirmando que, para os estudantes analisados, o programa de mobilidade acadêmica proporcionou um desenvolvimento pessoal maior do que o profissional, sendo que os objetivos de vivenciar experiências pessoais eram mais relevantes quando comparados aos objetivos de agregar habilidades profissionais.

A seguir, analisam-se as variáveis anteriores de acordo com o gênero.

\section{ANÁLISE DAS VARIÁVEIS EM RELAÇÃO AO GÊNERO}

Nesta seção, apresentam-se as variáveis que apresentaram diferenças estatisticamente significativas em relação ao gênero dos estudantes que realizaram mobilidade acadêmica, calculadas a partir do teste estatístico de Mann-Whitney, demonstradas na Tabela 3.

\section{Tabela 3 - Variáveis x gênero}

\begin{tabular}{|c|c|c|c|c|c|c|c|c|c|}
\hline & \multicolumn{6}{|c|}{$\begin{array}{l}\text { Objetivos que motivaram o investimento no programa de } \\
\text { intercâmbio }\end{array}$} & \multirow{2}{*}{\multicolumn{3}{|c|}{$\begin{array}{c}\begin{array}{c}\text { Razões que motivaram a } \\
\text { escolha do país de destino do } \\
\text { intercâmbio }\end{array} \\
\begin{array}{c}\text { Ter como língua oficial o } \\
\text { inglês }\end{array}\end{array}$}} \\
\hline & \multicolumn{3}{|c|}{ Vicência pessoal } & \multicolumn{3}{|c|}{ Conhecer outro país } & & & \\
\hline Gênero & $\mathrm{N}$ & $\begin{array}{l}\text { Posto } \\
\text { Médio }\end{array}$ & Sig. & $\mathrm{N}$ & $\begin{array}{l}\text { Posto } \\
\text { Médio }\end{array}$ & Sig. & $\mathrm{N}$ & $\begin{array}{l}\text { Posto } \\
\text { Médio }\end{array}$ & Sig. \\
\hline Masculino & 17 & 25,74 & \multirow{2}{*}{0,05} & 17 & 24,88 & \multirow{2}{*}{0,05} & 17 & 37,62 & \multirow{2}{*}{0,09} \\
\hline Feminino & 43 & 32,38 & & 43 & 32,72 & & 43 & 27,69 & \\
\hline
\end{tabular}

Fonte: Elaboração dos autores. 
Quando analisadas as variáveis a partir do gênero, percebe-se que 3 (três) delas apresentaram nível de significância $\mathrm{p}$ valor $<0,05$, ou seja, apresentaram diferença estatisticamente significativa entre o grupo masculino e o grupo feminino.

As variáveis que representam os objetivos que motivaram o investimento no programa de intercâmbio 'vivência pessoal' e 'conhecer outro país' apresentam postos médios maiores para o gênero feminino quando comparado ao gênero masculino, ou seja, para as estudantes do gênero feminino que realizaram programa de intercâmbio pela instituição analisada, a experiência de vida que seria proporcionada e o desejo de conhecer um país diferente foi mais determinante para a realização da mobilidade acadêmica do que para os estudantes do gênero masculino.

De forma contrária, a razão 'ter como língua oficial o inglês' como motivador para a escolha do país de destino do intercâmbio obteve postos médios maiores para o gênero masculino quando comparada ao gênero feminino, ou seja, para os estudantes do gênero masculino participantes de mobilidade acadêmica pela instituição, a escolha de um país de língua inglesa como idioma oficial foi mais determinante do que para as estudantes do gênero feminino.

A seguir, analisam-se as variáveis de acordo com a faixa etária.

\section{ANÁLISE DAS VARIÁVEIS EM RELAÇÃO A FAIXA ETÁRIA}

Nesta seção, apresentam-se as variáveis que apresentaram diferenças estatisticamente significativas no que diz respeito à faixa etária dos estudantes que realizaram mobilidade acadêmica, calculadas a partir do teste estatístico de kruskal-wallis, demonstradas na tabela 4.

\section{Tabela 4 - Variáveis x faixa etária}

\begin{tabular}{|c|c|c|c|c|c|c|}
\hline \multirow[b]{3}{*}{ Faixa Etária } & \multicolumn{3}{|c|}{$\begin{array}{l}\text { Objetivos que motivaram o } \\
\text { investimento no programa de intercâmbio }\end{array}$} & \multicolumn{3}{|c|}{$\begin{array}{l}\text { Resultados percebidos com o programa de } \\
\text { intercâmbio }\end{array}$} \\
\hline & \multicolumn{3}{|c|}{ Conhecer outra cultura } & \multicolumn{3}{|c|}{$\begin{array}{l}\text { Prospecção de alternativas de pós- } \\
\text { graduação }\end{array}$} \\
\hline & $\mathbf{N}$ & Posto Médio & Sig. & $\mathrm{N}$ & Posto Médio & Sig. \\
\hline Até 21 anos & 8 & 23,25 & \multirow{5}{*}{0,048} & 8 & 17,19 & \multirow{5}{*}{0,041} \\
\hline 22 ou 23 anos & 31 & 34,24 & & 31 & 30,26 & \\
\hline 24 ou 25 anos & 15 & 27,9 & & 15 & 37,47 & \\
\hline De 26 a 29 anos & 3 & 14,67 & & 3 & 21,17 & \\
\hline 30 anos ou mais & 3 & 40 & & 3 & 43 & \\
\hline
\end{tabular}

Fonte: Elaboração dos autores. 
Quando analisadas as variáveis a partir do gênero, percebe-se que 2 delas apresentaram nível de significância pvalor $<0,05$, ou seja, apresentaram diferença estatisticamente significativa entre as faixas etárias analisadas.

O objetivo de 'conhecer outra cultura' como motivador do investimento no programa de intercâmbio e a 'prospecção de alternativas de pós-graduação' como resultado percebido com o programa de intercâmbio apresentaram maiores postos médios para os estudantes com idade superior a trinta anos, ou seja, o objetivo de conhecer e se inserir em uma nova cultura e conseguir prospectar alternativas para capacitação profissional foram mais determinantes para o grupo de estudantes com idades mais elevadas quando comparadas às demais faixas etárias.

A seguir, analisam-se as variáveis de acordo com a renda familiar.

\section{ANÁLISE DAS VARIÁVEIS EM RELAÇÃO A RENDA FAMILIAR}

Nesta sessão, apresentam-se as variáveis que apresentaram diferenças estatisticamente significativas em relação à renda familiar dos estudantes que realizaram mobilidade acadêmica, calculadas a partir do teste estatístico de Kruskal-Wallis, demonstradas na Tabela 5.

\section{Tabela 5 - Variáveis $\mathrm{x}$ renda familiar}

\begin{tabular}{|c|c|c|c|c|c|c|}
\hline \multirow{3}{*}{ Renda Familiar } & \multirow{2}{*}{\multicolumn{3}{|c|}{$\begin{array}{c}\begin{array}{c}\text { Objetivos que motivaram o investimento no } \\
\text { programa de intercâmbio }\end{array} \\
\text { Conhecer outro país } \\
\end{array}$}} & \multirow{2}{*}{\multicolumn{3}{|c|}{$\begin{array}{l}\text { Razões que motivaram a escolha do país de } \\
\text { destino do intercâmbio } \\
\text { Facilidade de obtenção de visto }\end{array}$}} \\
\hline & & & & & & \\
\hline & $\mathrm{N}$ & Posto Médio & Sig. & $\mathrm{N}$ & Posto Médio & Sig. \\
\hline Até 1 s.m. & 1 & 40 & \multirow{5}{*}{0,045} & 1 & 53,5 & \multirow{5}{*}{0,044} \\
\hline De 1 a 3 s.m. & 11 & 31,23 & & 11 & 41,86 & \\
\hline De 3 a 5 s.m. & 21 & 36,02 & & 21 & 30,74 & \\
\hline De 5 a 15 s.m. & 18 & 28,94 & & 18 & 23,75 & \\
\hline Mais de 15 s.m. & 9 & 18,78 & & 9 & 27 & \\
\hline
\end{tabular}

Fonte: Elaboração dos autores.

Quando analisadas as variáveis a partir da renda familiar, percebe-se que duas delas apresentaram nível de significância p valor $<0,05$, ou seja, apresentaram diferença estatisticamente significativa entre as faixas de renda familiar analisadas.

O objetivo de 'conhecer outro país' como fator que motivou o investimento no programa de intercâmbio e a 'facilidade de obtenção de visto' como razão que motivou a escolha do país de destino do intercâmbio apresentaram maiores postos médios para os estudantes com renda familiar de até 5 salários mínimos (faixas 
até 1 s.m., de 1 a 3 s.m. e de 3 a 5 s.m.), ou seja, os estudantes de famílias menos abastadas consideraram a facilidade de emissão do visto como mais determinante para escolha do destino quando comparados àqueles pertencentes às famílias de menor renda; assim como o desejo de conhecer outro país ser considerado como um objetivo mais relevante pode ser atribuído ao fato de que estes acadêmicos, durante o período da graduação, teriam menor possibilidade de viajar ao exterior sem algum auxílio institucional.

A seguir, discorrem-se as considerações finais do trabalho.

\section{CONSIDERAÇÕES FINAIS}

Este estudo foi desenvolvido com o objetivo de identificar as motivações e contribuições da mobilidade acadêmica internacional para os alunos de uma instituição federal do Rio Grande do Sul. Por meio dos resultados apresentados, foi possível identificar os objetivos do intercâmbio, as razões de escolha do país e os resultados percebidos pelos estudantes que participaram de mobilidade acadêmica enquanto graduandos da Universidade Federal de Santa Maria.

Os objetivos que motivaram os estudantes da instituição analisada a optarem pela inserção em um programa de intercâmbio se devem, principalmente, a ter uma vivência pessoal, a conhecer uma cultura diferente, a ter a oportunidade de viajar para um país ainda não visitado, a questões relacionadas ao curso que estudam, a aperfeiçoar uma língua estrangeira e a aumentar a amplitude de oportunidades para aperfeiçoamento profissional. Contudo, percebeu-se que os objetivos mais relevantes que motivaram a realização de intercâmbio pelos estudantes investigados estão mais relacionados com experiências pessoais quando comparados a objetivos profissionais. Entre as razões que motivaram a escolha do país de destino do intercâmbio, percebeu-se que fatores relacionados à estrutura que o país de origem oferece; baixos custos, boa infraestrutura e instituições acadêmicas atraentes são razões significativas para a escolha do país de destino do estudante em mobilidade acadêmica da instituição analisada. Quanto aos resultados percebidos com o programa de intercâmbio, a análise indicou que a experiência pessoal pretendida com o programa de mobilidade acadêmica foi vivenciada de maneira que os resultados relacionados a essas competências pessoais foram mais significativos do que os resultados relacionados ao desenvolvimento profissional.

Quando analisadas a partir do gênero, constatou-se que, para as estudantes do gênero feminino que realizaram programa de intercâmbio pela instituição analisada, a experiência de vida que seria proporcionada e o desejo de conhecer um país diferente foi mais determinante para a realização da mobilidade acadêmica do que para os estudantes do gênero masculino. No entanto, para 
os estudantes do gênero masculino participantes de mobilidade acadêmica pela instituição, a escolha de um país de língua inglesa como idioma oficial foi mais determinante do que para as estudantes do gênero feminino. Notou-se, também, que o objetivo de conhecer e inserir-se em uma nova cultura e conseguir prospectar alternativas para capacitação profissional foram mais determinantes para o grupo de estudantes com idades mais elevadas quando comparados às demais faixas etárias. Além disso, percebeu-se que o desejo de conhecer outro país como objetivo para realização do intercâmbio e a facilidade de emissão do visto como razão para escolha do país foram mais determinantes para os estudantes de famílias menos abastadas quando comparados àqueles pertencentes às famílias de maior renda.

Ressalta-se, por fim, que esta pesquisa possui limitações. Por tratar-se de uma amostra por conveniência, os resultados aqui apresentados referem-se aos estudantes que participaram desta pesquisa, impossibilitando a inferência destes para os demais estudantes participantes de mobilidade acadêmica desta e de outras instituições. Como sugestão para trabalhos futuros, pode-se destacar que se busque ampliar o público-alvo, contemplando outras instituições de ensino.

\section{REFERENNCIAS}

AGUIAR, A. Estratégias educativas de internacionalização: uma revisão da literatura sociológica. Educação e Pesquisa, v.35, n.1, jan.-abr., p.67-79, 2009

ALMEIDA, A. M. F. Língua nacional, competência escolar e posição social. In: Almeida, Ana Maria F. et alii. Circulação internacional e formação intelectual das elites brasileiras. Campinas: Editora Unicamp, p.29-46, 2004

CASTRO, A. A.; NETO, A. C. O ensino superior: a mobilidade estudantil como estratégia de internacionalização na América Latina. Revista Lusófona de Educação, núm. 21, pp. 69-96. out. 2012

DOUEK, A.;ZYLBERSTAJN, A. Intercâmbio:influênciana empregabilidade do administrador. 2007, Monografia (Graduação em Administração de Empresas) - na Escola Superior de Propaganda e Marketing, São Paulo, 2007.

FILIPPETTI, A. Action extérieure de l'État rayonnement culturel et scientifique. France: Assemblée Netionale, n. 277, 11, 2007. 
GuimarÃeS; TADEuCCI, M. DE. S.; OLIVEIRA, DE. A. L. Estudo bibliométrico em gestão intercultural, internacionalização e mobilidade acadêmica: foco no ensino superior. Janus, Lorena, n.17, Jan./Jun., 2013. p. 55-65.

HARFI, M. Attractivité pour les étudiants étragers et potentiel de la recherche en France. Le quatre pages, n.2, 15/07/2004. HARFI, Mohamed; MATHIEU, Claude. Mobilité internationale et attractivité des étudia Higher Education, $n^{\circ} 41$, p. 415-441, 2001.

HARFI, M. Attractivité pour les étudiants étragers et potentiel de la recherche en France. Le quatre pages, n.2, 15/07/2004.

HARFI, M; MATHIEU, C. Mobilité internationale et attractivité des étudiants et des chercheurs. Centre d'Analyse Stratégique: Horizons Stratégiques, n.1, juillet 2006

IANNI, O. A sociedade global. Rio de Janeiro: Civilização Brasileira, $12^{a}$ ed., 2005

LARSEN, K.; VICENT-LANCRIN, S. Le commerce international de services d'éducation: Est-il bon? Est-il méchant? Politiques et gestion de l'enseignement supérieur. 14 (3), déc. 2002

LIMA, M. C.; MARANHÃO, C. M. do S. de A. (2009). O sistema de educação superior mundial: entre a internacionalização ativa e passiva. Revista Avaliação, Campinas; Sorocaba, SP, v. 14, n. 3, p. 583-610, nov. 2009.

LIMA, M. C.; RIEGEL, V. Motivações da Mobilidade Estudantil entre os estudantes do Curso de Administração. Guavira Letras, n. 10, Jan.-Jul. 2010.

MUÑOZ, M. C. Políticas francesas de acolhimento dos estudantes estrangeiros. (1970-2002) In: ALMEIDA, Ana Maria F. et al. Circulação internacional e formação intelectual das elites brasileira. Campinas: Editora da Unicamp, p.188-212, 2004.

NOGUEIRA, M A; AGUIAR, A. M. S; RAMOS, V. C. C. Fronteiras desafiadas: a internacionalização das experiências escolares. Educação e Sociedade, v. 29, n. 103, p. 355-376, 2008. 
NOGUEIRA, M. A. Viagens de estudo ao exterior: as experiências de filhos de empresários. In: Almeida, Ana Maria F. et alli. Circulação internacional e formação intelectual das elites brasileiras. Campinas: Editora Unicamp, p. 4763, 2004.

PINSONNEAULT, A.; KRAEMER, K. L. Survey research in management informations systems: an assessement. Journal of Management Information Systems, v.10, n. 2, p. 75-106, 1993

PRADO, C. L. Um aspecto do estudo de línguas estrangeiras no Brasil: os intercâmbios. In: Almeida, Ana Maria F. et alli. Circulação internacional e formação intelectual das elites brasileiras. Campinas: Editora Unicamp, 2004, p.64-84.

RICHARDSON, R. J. Pesquisa social: métodos e técnicas. São Paulo: Atlas, 1999.

ROMÊ, J.R.M. Higher Education in Latin America' in Higher Education in Europe, 28,1, 41-49. 2003

STALLIVIERI, L. Estratégias de internacionalização das Universidades Brasileiras. Caxias do Sul: Educs, 2004.

TRIVINOS, A. N. S. Introdução a pesquisa em ciências sociais: a pesquisa qualitativa em educação. São Paulo: Atlas, 1987

UNESCO. Educação: Um tesouro a descobrir. Relatório para a UNESCO da Comissão Internacional sobre Educação para o século XXI. São Paulo, Cortez; Brasília: MEC UNESCO, 2006

UNESCO/Institut de Statistique. Recueil des données mondiales sur l'éducation: statistiques comparées sur l'éducation dans le monde, 2003; 2004; 2005; 2006; 2007

VAN DAMME, D. Quality issues in the internationalization of higher education. Higher Education, nº 41, p. 415-441, 2001. 
ANA PAULA PERLIN possui Doutorado em andamento em Administração, Mestrado em Administração e Especialização em Gestão Pública pela Universidade Federal de Santa Maria, UFSM, Brasil. Graduação em Administração pela Universidade Federal de Santa Maria, UFSM, Brasil. Com período sanduíche em Universidade do Minho. E-mail: anapaulaperlin@gmail.com

GABRIELA ROSSATO possui Mestrado em andamento em Administração. Universidade Federal de Santa Maria, UFSM, Brasil. Graduação em Administração. Universidade Federal de Santa Maria, UFSM, Brasil. E-mail: gabi. rossato@hotmail.com

FRANCIES DIEGO MOTKE possui Doutorado em andamento em Administração, Mestrado em Administração e graduação em Administração pela Universidade Federal de Santa Maria, UFSM, Brasil. E-mail: francies@hotmail.com

CLANDIA MAFFINI GOMES possui Doutorado em Administração pela Universidade de São Paulo, USP, Brasil, com período sanduíche em Boccony University. Mestrado em Administração, Especialização em Gestão Empresarial e Graduação em Administração de Empresas pela Universidade Federal de Santa Maria, UFSM, Brasil. E-mail: clandia@hotmail.com

JORDANA MARQUES KNEIPP possui Doutorado em Administração pela Universidade Federal de Santa Maria, UFSM, Brasil com período sanduíche em Universidad de Vigo. Mestrado em Administração pela Universidade Federal de Santa Maria, UFSM, Brasil. Graduação em Administração pela Universidade Federal de Pelotas, UFPEL, Brasil. Curso técnico/profissionalizante em Técnico em Contabilidade. Instituto Estadual de Educação Dinarte Ribeiro, IEEDR, Brasil.E-mail: jordana@hotmail.com 